\title{
Asymptomatic fatal post-lobectomy hemopericardium
}

\author{
C. Kotoulas, S. Meraj, N.J. Odom
}

\begin{abstract}
Asymptomatic fatal post-lobectomy hemopericardium. C. Kotoulas, S. Meraj, N.J. Odom.

We report a case of an asymptomatic post-lobectomy hemopericardium in a female who died suddenly at day two post surgery. Autopsy revealed no pathologic findings,
\end{abstract}

but $250 \mathrm{ml}$ of blood and clots in the pericardium and a non-significant injury to the epicardial fat overlying the circumflex artery territory.

Monaldi Arch Chest Dis 2008; 69: 3, 134-136.

Keywords: Haemopericardium, Major lung resection, Lung cancer, Surgery.

Department of Cardiothoracic Surgery, Manchester Royal Infirmary, Manchester, UK.

Correspondence: Christophoros Kotoulas, MD, PhD, FETCS; Kifissias 38, Ampelokipoi, GR-11526, Greece, e-mail: chrkotoulas@hol.gr

\section{Introduction}

Haemopericardium is a common complication in cardiac surgery, but extremely rare in lung or esophageal surgery. Arrhythmias, pulmonary embolism and myocardial infarction are the most common cardiovascular complications after major lung resection, especially in the cases with intrapericardial ligation of the vessels $[1,2]$.

We report herein, a case of an asymptomatic fatal haemopericardium after major lung resection, discussing the possible involved mechanism and reviewing the few reported cases.
On day 1 , the basal drain was removed, having no air leak (figure 2). Following this, she had a short episode of hypotension (MAP $65 \mathrm{~mm} \mathrm{Hg}$ ) that was improved with a minute dose of noradrenaline and colloids and discontinuation of epidural, as it was thought to be due to epidural. A few hours later, noradrenaline was tapered off. All the monitoring factors remained stable. On day 2, she was cardiovascularly stable, and the apical chest drain was removed. Her chest X-ray revealed no abnormal findings and she was transferred to the ward (figure 3). She remained well and continued to mobilise herself. Four hours later, the patient was

\section{Case report}

A 70 year-old lady was admitted to our department due to lung cancer. A computed tomography scan showed a $2-\mathrm{cm}$ nodule in the left upper lobe and PET-scan certified that there was no metastatic lesion. After a pre-operative evaluation she underwent an uneventful left standard upper lobectomy through a posterolateral thoracotomy. During the induction of anaesthesia, an epidural catheter was inserted to manage the post-operative pain.

Post-operatively, she was transferred to the High Dependency Unit where she was cardiovascularly stable with a mean arterial pressure (MAP) more than $75 \mathrm{~mm}$ $\mathrm{Hg}$ and a good urine output. Antero-posterior chest $\mathrm{x}$-ray in a semi-supine position showed full expansion of the left lung without other abnormal findings (figure 1).

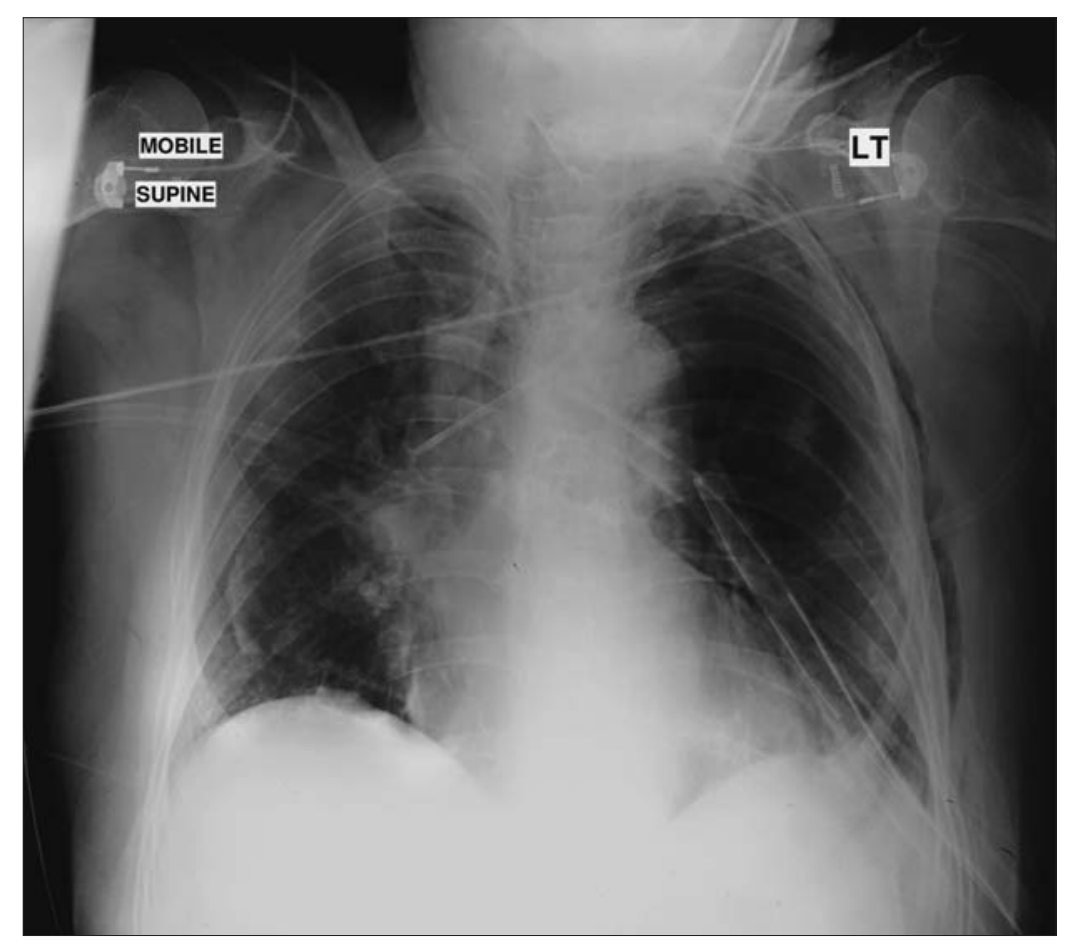

Fig. 1. - Mobile chest X-ray (anteroposterior) shows the full expansion of the left lung and the two chest drains. 


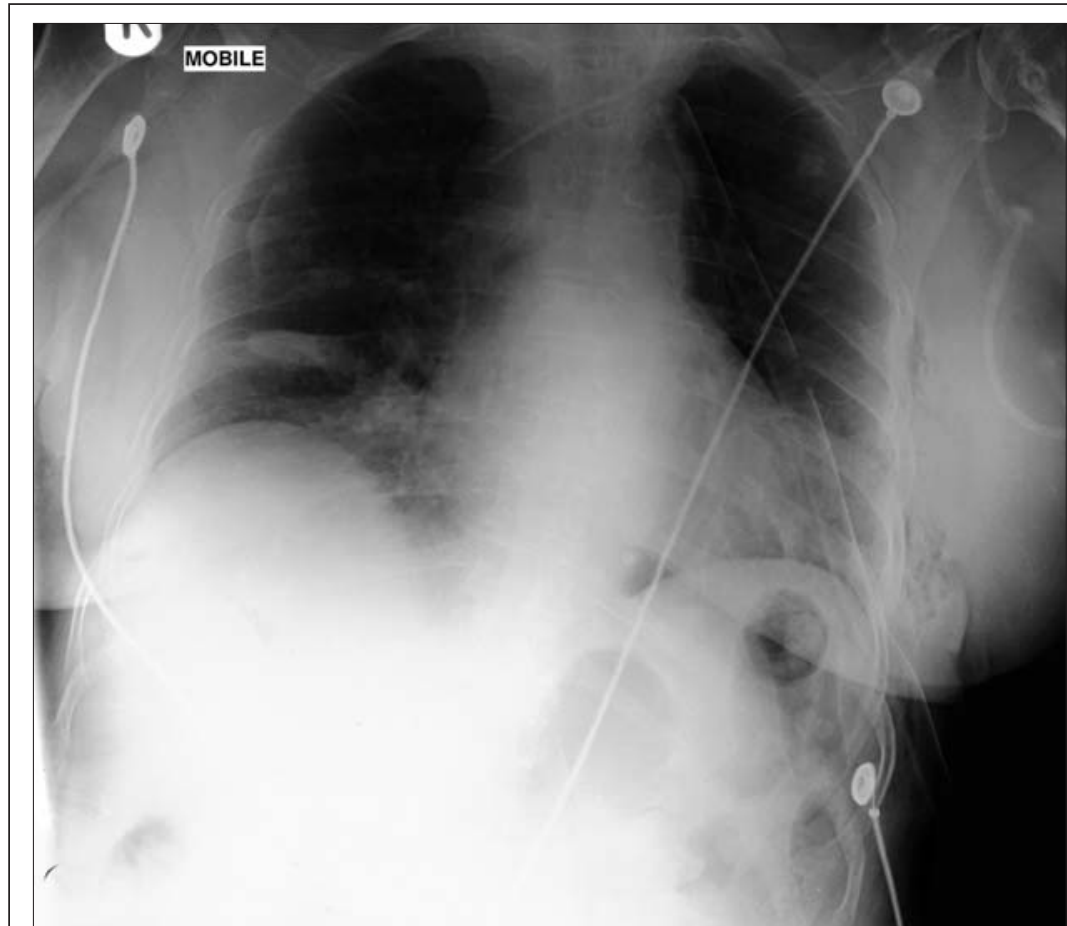

Fig. 2. - Mobile chest x-ray (anteroposterior) shows the full expansion of the lung and the apical chest drain.

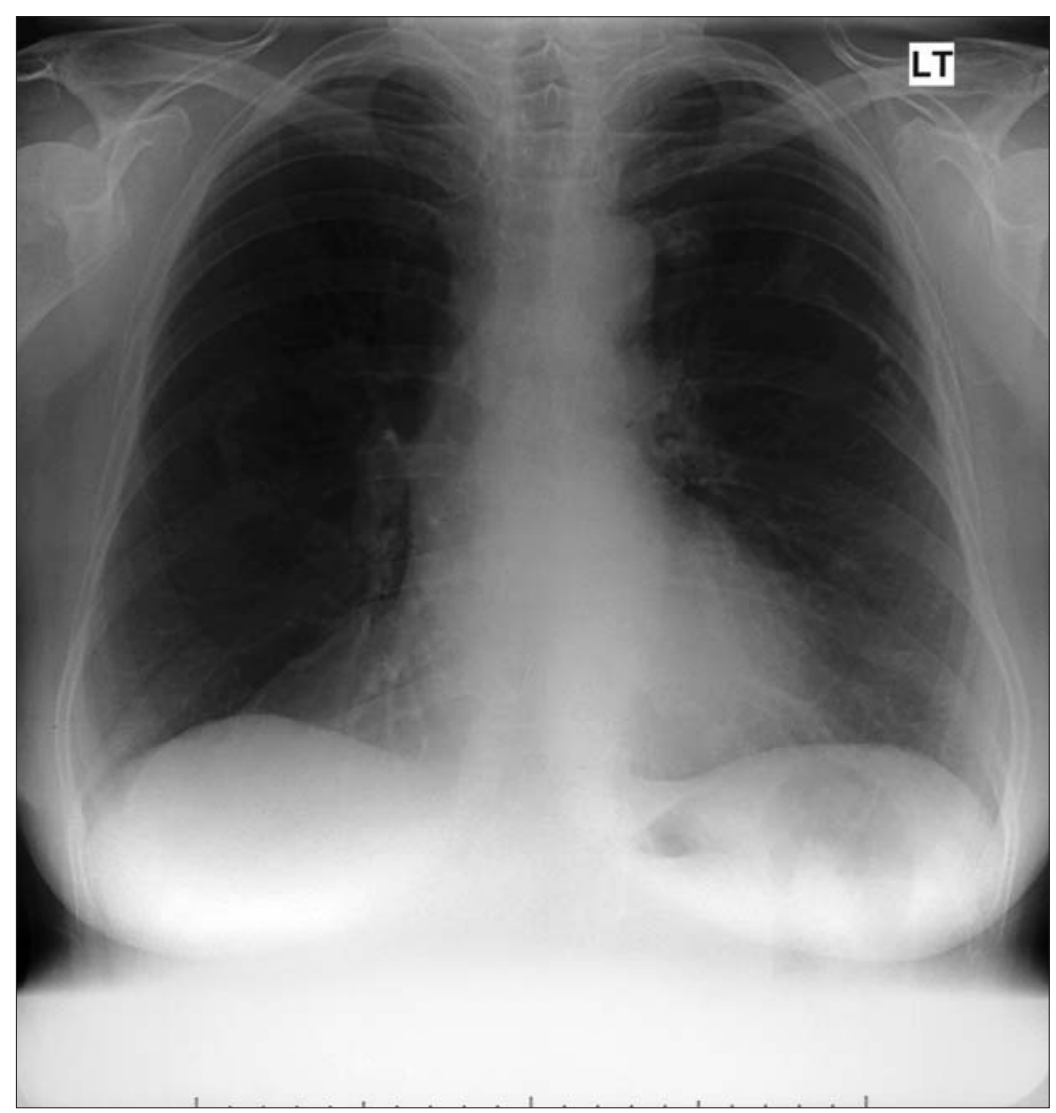

Fig. 3. - Chest $\mathrm{x}$-ray in an erect position (posteroanterior) shows full expansion of the lung with no signs for existence of pericardial effusion.

found unresponsive in the bed with asystolic cardiac arrest. A chest drain was inserted but no air or fluid was found in the pleural cavity. Despite the resuscitation, she passed away.

Autopsy showed approximately $250 \mathrm{mls}$ of fresh blood and blood clot surrounding the heart, suggestive of cardiac tamponade. There was an area $2 \times 0.5 \mathrm{~cm}$ of haemorrhage in the epicardial fat, situated over the left lateral branch of the left circumflex coronary artery. There was no finding of myocardial infarction. There were tied off vessels extrapericardially at the left hilum and $50 \mathrm{mls}$ of blood stained fluid in left pleural cavity. No air or fluid was found in the right pleural cavity. There was no evidence of pulmonary embolism or stroke.

\section{Discusssion}

The major causes attributed to mortality during the first days after thoracic operations are pulmonary embolism, myocardial infarction, massive cerebrovascular event and tension pneumothorax. Cardiac tamponade is a very rare complication after thoracic surgery, as only a few cases with different involved mechanisms have been reported in the past.

Morimoto et al, first, described a case of cardiac tamponade and hemothorax following rupture of the ascending aorta which occurred on the 13th post-operative day following a right upper lobectomy with mediastinal lymph node dissection for lung cancer. The operative findings suggested a laceration on the lateral wall of the ascending aorta; however, injuries of the pericardium from the diathermy during the mediastinal lymph node dissection could not be excluded as a possible cause [3].

Tovar reported a case of intraoperative cardiac tamponade during a left lower lobectomy, where the inferior pulmonary vein had slipped off the clamp during its ligation, bleeding freely into the pericardial sac. The acute tamponade was recognized immediately and corrected without catastrophic consequences [4].

Furthermore, Mc Clean et al described a case of cardiac tamponade following a right upper lobectomy, due to an aberrant right bronchial artery, that coming off the intra-pericardial portion of the aorta. The vessel was retracted into the pericardial sac after being cauterised and subsequently bled [5].

Pillai et al reported a case of cardiac tamponade due to partial retraction of the superior pulmonary vein intrapericardially and suture line oozing in it [1].

Our case is only that the patient was asymptomatic and the reason of the death discovered dur- 
ing the autopsy. The only postperative sign that could be attributed to cardiac tamponade was low blood pressure. However, it was thought to be a thoracic epidural consequence, as it improved on giving inotropes and fluids and there was no further drop in blood pressure after the removal of the epidural catheter. Additionally, the diagnosis of the tension pneumothorax was excluded as a chest drain was inserted following the cardiac arrest but no blood or air was found in the pleural cavity.

The entire operative course was uneventful. The superior pulmonary vein was ligated extrapericardially, as well as the branches of the pulmonary artery for the upper lobe Autopsy showed mediastinal pleura and pericardium did not have any puncture marks. Although, on autopsy there was an area of disruption and haemorrhage situated over the left lateral branch of the left circumflex coronary artery, the overlying pericardium was intact. As there was no obvious reason, we speculate that there was a blind needle stick injury of the territory of circumflex coronary artery when the superior pulmonary vein was tied. As a consequence, the intrapericardial blood and clots as well as the hyperinflation of the right lung and mediastinal deviation resulted in acute pressure to the right car- diac chambers and the cardiac arrest upon mobilisation of the patient

In conclusion, we report a rare case of an asymptomatic, but fatal cardiac tamponade following left upper lobectomy. We emphasise the need for detailed investigation with echocardiography in cases of unexplained hypotension after lung resection, as well as the fact that the pulmonary vein ligation must be meticulous in order to prevent such a rare, but in most cases, fatal, complication.

\section{References}

1. Pillai JB, Barnard S. Cardiac tamponade: a rare complication after pulmonary lobectomy. Interact Cardiovasc Thorac Surg 2003; 2: 657-659.

2. Mizuguchi Y, Takeda S, Miyashita M, et al. A case of cardiac tamponade following esophageal resection. $J$ Anesth 2005; 19: 249-251.

3. Morimoto M, Ohashi M, Nobara H. Rupture of the ascending aorta after surgical resection for lung cancer. Jpn J Surg 1991; 21: 474-479.

4. Tovar EA. Pulmonary resection complicated by abrupt pericardial tamponade. Ann Thorac Surg 1995; 60: 1864.

5. McLean R, Parandian B, Nam M. Pericardial tamponade: an unusual complication of lobectomy for lung cancer. Ann Thorac Surg 1999; 67: 545-546.

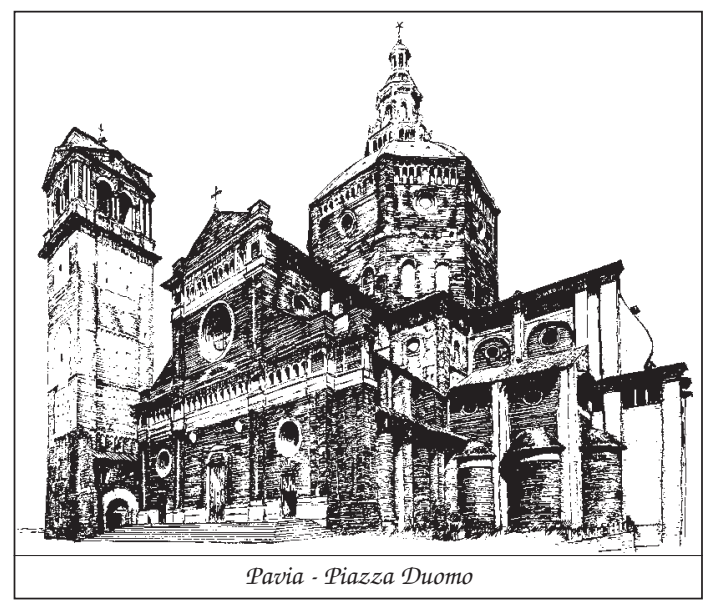

